\title{
Surface-wave Bragg resonators for terahertz frequency range
}

\author{
A.M. Malkin, N.S. Ginzburg, S.E. Fil'chenkov, A.S. Sergeev, and V.Yu. Zaslavsky \\ Institute of Applied Physics RAS, Nizhny Novgorod, Russia, malkin@appl.sci-nnov.ru
}

Surface electromagnetic waves confined at the interface of metal and dielectric have been widely studied [1] since they possess a number of unique features making them useful for various applications, including antenna applications Raman scattering, etc. Since the evanescent waves are decelerated, Cherenkov devices based on interaction of electron beams with surface modes of the corrugated structures is of special interest in the short wavelength radiation bands, including the terahertz band [2]. These devices allow to solve the problem of both longitudinal and transverse mode selection in oversized electrodynamical systems.

In this paper, we consider a periodically corrugated section of a metallic plate as a surface-wave resonator (see Fig. 1). For the limiting case of shallow corrugation, we obtain explicitly the equations of wave coupling with Ohmic losses taken into account and derive an integral equation describing the eigenmodes. Based on this equation, we find the optimum values of the corrugation depth providing the maximum $\mathrm{Q}$ factor of the fundamental mode.

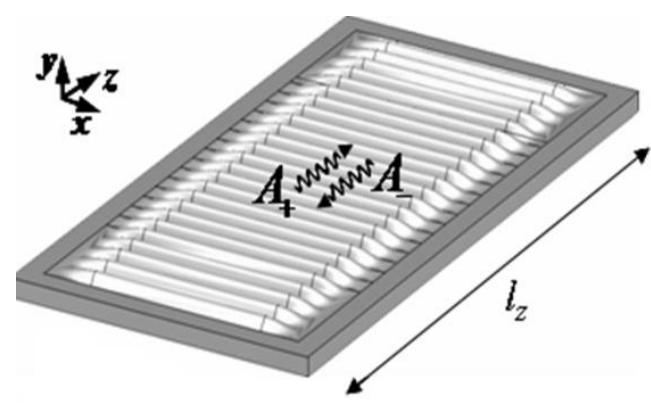

Fig. 1. Surface-wave resonator

In the case when the amplitude of sinusoidal corrugation $b(z)=\left(b_{0} / 2\right) \cos \bar{h} z$ is small in the scale of wavelength and of the structure period $d$ the electromagnetic field of the surface mode existing near the 1D corrugated metal plate can be presented as a sum of two quasioptical wavebeams with slowly varying amplitudes $A_{ \pm}$ which can be characterized by the magnetic field

$$
H_{x}=\operatorname{Re}\left[A_{+}(z, y, t) e^{i\left(\omega_{0} t-h_{0} z\right)}+A_{-}(z, y, t) e^{i\left(\omega t+h_{0} z\right)}\right] \text {, }
$$

where $h_{0}=\bar{h} / 2=\pi / d, \omega_{0}=c h_{0}$ is the Bragg frequency. Propagation of electromagnetic field in the vicinity of the Bragg resonance can be described by a system of coupled parabolic wave-beam equations which take into account the diffraction in y direction [2],

$$
\pm \frac{\partial A_{ \pm}}{\partial z}+\frac{\partial A_{ \pm}}{c \partial t}+\sigma \delta(y) A_{ \pm}+\frac{i}{\bar{h}} \frac{\partial^{2} A_{ \pm}}{\partial y^{2}}=i \alpha \delta(y) A_{\mp}
$$

At the edges of the corrugation we set non-reflection boundary conditions: $A_{+}(z=0)=0, A_{-}\left(z=l_{z}\right)=0$. Here $\alpha=\bar{h} b_{0} / 8$ is the coupling parameter, $\delta(y)$ is the delta function. Parameter $\sigma=\sqrt{i / 2} k d_{\text {skin }}$ describes the Ohmic losses in the metal corresponding to the Leontovich boundary condition, $d_{\text {skin }}$ is the skin depth of the metal.

Equations (1) applied to a system infinite in $z$ direction lead to the existence of a surface wave $A_{+} \quad e^{i \Gamma z-g_{ \pm} y+i \Omega t}$ with a dispersion law which in the lossless case can be put down as

$$
\left(\frac{\Omega}{c}\right)^{2}-\Gamma^{2}=\bar{h}^{2} \alpha^{4}
$$

Dispersion characteristic corresponding to (2) is shown in the lower part of Fig. 2.

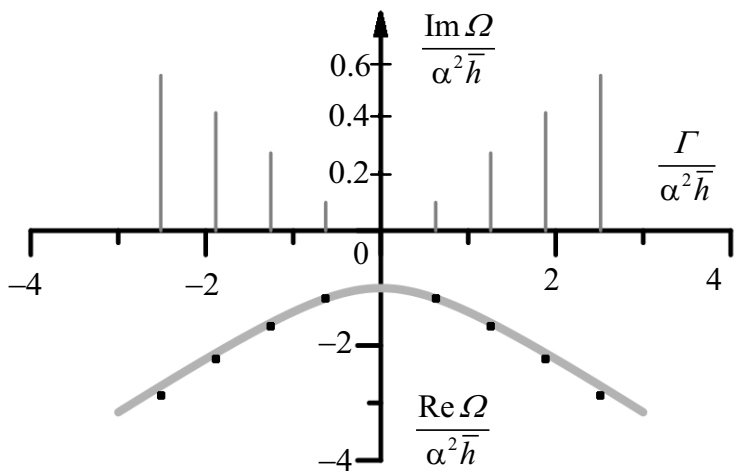

Fig. 2. Dispersion curve of the surface wave. Dots mark the surface-wave resonator modes at $\alpha^{2} \bar{h} l_{z}=5$, lines in the upper part indicate the losses of these modes

In the case when the length $l_{z}$ of the structure is finite, a set of longitudinal modes can be found. Seeking modes of (1) at an eigenfrequency of $\Omega$, and using the Fourier transform, one can obtain an integral equation equivalent to system (1):

$$
\begin{aligned}
& \hat{A}_{+}(0, z)= \\
& =\sqrt{\frac{\bar{h}}{\pi i}} \int_{0}^{z} \frac{ \pm \alpha\left(z^{\prime}\right) \hat{A}_{+}\left(0, l_{z}-z^{\prime}\right)+i \sigma \hat{A}_{+}\left(0, z^{\prime}\right)}{\sqrt{z-z^{\prime}}} e^{-i \Omega\left(z-z^{\prime}\right)} d z^{\prime} .
\end{aligned}
$$

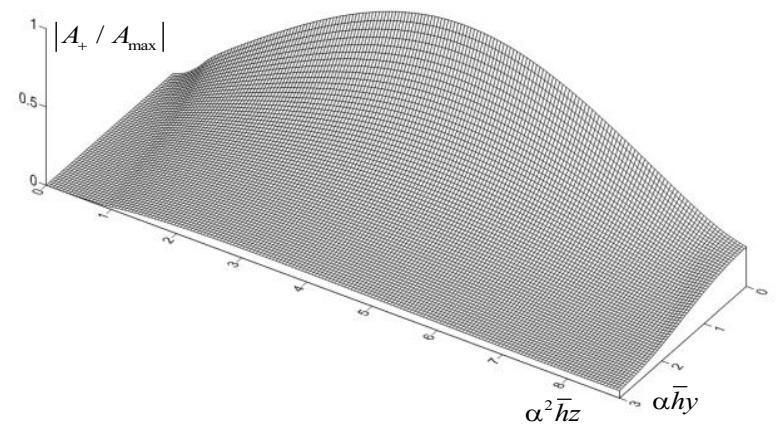

Fig. 3. Fundamental mode structure at $\alpha^{2} \bar{h} l_{z}=8.8$ 
Equation (3) possesses an infinite discrete eigenmode spectrum. Frequencies and spatial profiles of eigenmodes can be found numerically. Real parts of the eigenfrequencies of the first several modes are marked with dots close to the dispersion in lower part of Fig.2 for $\alpha^{2} \bar{h} l_{z}=5$ while the imaginary parts of these modes are shown as vertical lines in the upper part of the same figure. It can be seen that the $\operatorname{Im} \Omega$ increases almost linearly. Quality factor $Q=\omega_{0}(2 \operatorname{Im} \Omega)^{-1}$ of the fundamental mode is about 3 times higher than that the next (second mode). It demonstrates the high selective properties of surface wave resonator. Spatial distribution of the $A_{+}$field for the lowest (fundamental) mode is shown in Fig. 3.

Making use of the fact that according to the exact solution of (3) the longitudinal field structure of the mode tends to sine shape with increasing parameter $\alpha^{2} \bar{h} l_{z}$ (see Fig. 3), an asymptotic formulas for $\mathrm{Q}$ factors can be obtained in the following form:

$$
\begin{gathered}
Q=\frac{1}{Q_{d i f f}^{-1}+Q_{O h m}^{-1}}, \\
Q_{\text {diff }}=\frac{\alpha^{4} \bar{h}^{3} l_{z}^{3}}{5 \pi^{2}} ; Q_{O h m}=\frac{2}{\alpha \operatorname{Re} \sigma} .
\end{gathered}
$$

Note that the diffraction $\mathrm{Q}$ factor increases with the length of corrugated section while the Ohmic Q remains constant. At small $l_{z}$, the diffraction quality prevails, $Q \approx Q_{\text {diff }}<<Q_{O h m}$. With increase of the corrugation length, these two terms become comparable and the total quality factor tends to saturation at $Q \approx Q_{o h m}$. These dependences are shown in Fig.4, based both on the asymptotic formulas (dashed line) and on the exact solution of the integral equation (3) for three values of $\sigma / \alpha$ parameter.

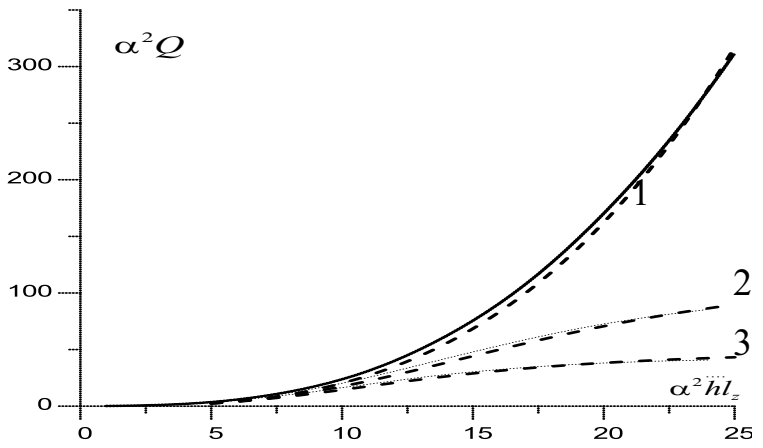

Fig. 4. Dependence of Q-factor on the $\alpha^{2} \bar{h} l_{z}$ parameter, 1) $\sigma / \alpha=0,2) \sigma / \alpha=0.1,3) \sigma / \alpha=0.25$

It is interesting to note that the two terms in (4) demonstrate different behavior at varying parameter $\alpha$ that is proportional to the corrugation depth $b_{0}$. While the diffraction term rises as $Q_{\text {diff }} \sim \alpha^{4}$, the Ohmic losses decrease as $Q_{O h m} \quad \alpha^{-1}$. This can be explained in a simple way: while the wavebeam coupling rises, the modes become more confined to the corrugation thus reducing the diffraction losses; at the same time, the less the effective 'volume' of the mode is, the higher is the influence of the Ohmic losses. Total Q factor would have a maximum in its $\alpha$ dependence for each $L$. This maximum can be found from expressions (4) as

$$
Q_{\max }=\left(\frac{2^{4}}{5^{6} \pi^{2}} \frac{l_{z}^{3}}{\bar{h} d_{\text {skin }}^{4}}\right)^{1 / 5} \approx 0.16 \frac{l_{z}^{3 / 5}}{\overline{h^{1 / 5}} d_{\text {skin }}^{4 / 5}}
$$

reached at

$$
\alpha_{\text {opt }}=\left(\frac{10 \pi^{2}}{\bar{h}^{4} l_{z}^{3} d_{\text {skin }}}\right)^{1 / 5} \approx \frac{3.97}{\bar{h}^{4 / 5} l_{z}^{3 / 5} d_{\text {skin }}^{1 / 5}} .
$$

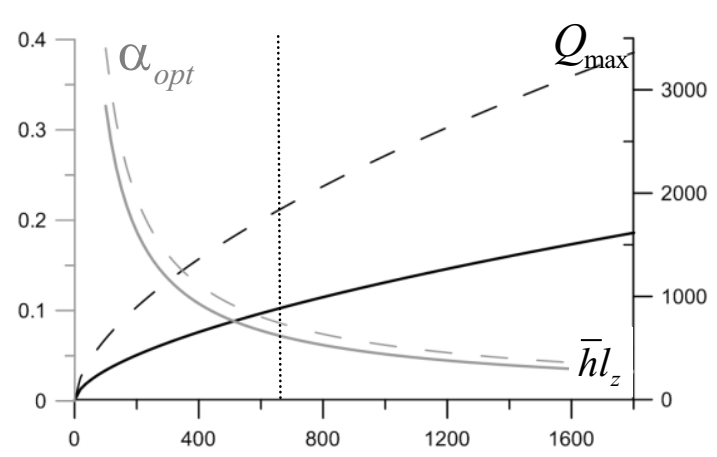

Fig. 5. Optimal $\alpha$ (gray) and maximum $Q$ (black) and vs length of $1 \mathrm{THz}$ resonator. Solid lines correspond to copper at $300 \mathrm{~K}$, dashed lines correspond to copper at $77 \mathrm{~K}$

The optimum $Q$ and $\alpha$ values vs. length of the resonator are depicted in Fig.5 for a structure with a Bragg frequency of about $1 \mathrm{THz}(\mathrm{d}=150 \mu \mathrm{m})$ made of copper at normal (solid lines, $d_{\text {skin }} \approx 0.065 \mu \mathrm{m}$ ) and liquid nitrogen (dashed lines, $d_{\text {skin }} \approx 0.025 \mu \mathrm{m}$ ) temperatures. According to (5), in this parameter area the small corrugation approximation is validated for structures of several dozen periods or longer. For instance, for a structure with a length of 100 periods ( $\bar{h} l_{z} \approx 630$, see dotted line in Fig. 5), a corrugation depth of $1 / 10$ wavelength provides an optimal Q factor of about 1000 for copper at normal temperature. For a copper structure at nitrogen temperature Q factor can reach 2000 for a slightly deeper corrugation.

Note in conclusion that similar analysis of surfacewave resonators with $2 \mathrm{D}$ corrugation can also be undertaken. Such structures provide mode selection in all three dimensions [3]. Using the time-domain simulation we can find the fundamental mode, its spatial profile, eigenfrequency, and $\mathrm{Q}$ factor. 00890 .

This work was supported by RFBR grant No. 16-02-

\section{References}

1. W. L. Barnes, A. Dereux, and T.W. Ebbesen. Surface plasmon subwavelength optics // Nature, v. 424, pp. 824-830, 2003.

2. N.S. Ginzburg, A.M. Malkin, A.S. Sergeev, V.Yu. Zaslavsky, Quasi-optical theory of relativistic submillimeter surfacewave oscillators // Appl. Phys. Lett. 2011, v. 99, p. 121505.

3. N.S. Ginzburg, A.M. Malkin, A.S. Sergeev, V.Yu. Zaslavsky. Powerful surface-wave oscillators with two-dimensional periodic structures Appl. Phys. Lett. 2012, v. 100, p. 143510. 